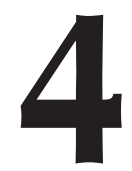

\title{
INCLUSIÓN EDUCATIVA COMPARADA EN UNESCO Y OCDE DESDE LA CARTOGRAFÍA SOCIAL
}

\section{(COMPARATIVE EDUCATIONAL INCLUSION IN UNESCO AND OECD FROM SOCIAL CARTOGRAPHY)}

María Jesús Martínez-Usarralde

Universitat de València

DOI: $10.5944 / e d u c X X 1.26444$

\section{Cómo referenciar este artículo/How to reference this article:}

Martínez-Usarralde, M.J. (2021). Inclusión educativa comparada en UNESCO y OCDE desde la cartografía social. Educación XX1, 24(1), 93-115, http://doi.org/10.5944/educXX1.26444

Martínez-Usarralde, M.J. (2021). Comparative educational inclusion in UNESCO and OECD from social cartography. Educación XX1, 24(1), 93-115, http://doi.org/10.5944/educXX1.26444

\section{RESUMEN}

La inclusión como principio tanto pedagógico como parte de la política socioeducativa se ha asentado a nivel transnacional. La aportación del presente artículo consiste en demostrar cómo, a pesar de los discursos que hablan de homogeneización y de isomorfismos en los principios sociopolíticos de la educación debido a las directrices pergeñadas desde los organismos internacionales, se detectan divergencias que fidelizan a cada uno de ellos con modelos teóricos irreconciliables. Bajo esta aspiración se plantea una metodología cualitativa en dos fases: en la primera se acomete el estudio de caso a partir de los informes publicados entre 1994 y 2019, a fin de escrutar el discurso que sobre la inclusión educativa han mantenido dos organismos idiosincrásicos como son OCDE y UNESCO en materia de inclusión educativa, detectando con ello las convergencias y las 
divergencias manifiestas en su retórica discursiva. En la segunda, se adopta la cartografía social, técnica usualmente utilizada en educación comparada, para evidenciar las ideas-fuerza que se han seleccionado de aquellos textos que construyen el debate: la OCDE y su visión de equidad que limita y condiciona a la inclusión y la UNESCO y su enfoque de modelo de derechos desde la justicia social. En los resultados se identifican las posiciones polares intertextuales que existen en los ejes (la teoría de desarrollo económico redistributivo y la teoría sociocrítica, respectivamente) de las que se deriva su propia idiosincrasia. Finalmente, en las conclusiones, se reconocen las comunidades textuales que comparten la forma de ver y comunicar la realidad (su visión de ambas de la responsabilidad que las instituciones escolares tienen de acuerdo con una conceptualización más social de la, primero discapacidad y, ahora, inclusión educativa).

\section{PALABRAS CLAVE}

Inclusión educativa, organismos internacionales, UNESCO, OCDE, cartografía social

\section{ABSTRACT}

Inclusion as both a pedagogical principle and part of socio-educational policy has become established at the transnational level. The contribution of this article consists of demonstrating how, despite the discourses that speak of homogenization and isomorphisms in the socio-political principles of education due to the guidelines established by international organizations, divergences are detected that make each of them loyal to irreconcilable theoretical models. Beyond this aspiration, a qualitative methodology is proposed in two phases: in the first, the case study is undertaken on the basis of reports published between 1994 and 2019, in order to scrutinize the discourse on educational inclusion maintained by two idiosyncratic organisms, OECD and UNESCO, in terms of educational inclusion, thereby detecting the convergences and divergences manifested in their discursive rhetoric. In the second, social cartography, a technique usually used in comparative education, is adopted in order to highlight the ideas that have been selected from those texts that construct the debate: the OECD and its vision of equity that limits and conditions inclusion, and UNESCO and its approach as a model of rights based on social justice. The results identify the intertextual polar positions that exist in the axes (the theory of redistributive economic development and the socio-critical theory, respectively) from which their own idiosyncrasies are derived. Finally, in the conclusions, the textual communities that share the way of seeing and communicating 
reality are recognized (their vision of both of the responsibility that school institutions have according to a more social conceptualization of, first disability and, now, educational inclusion).

\section{KEY WORDS}

Educational inclusion, international organizations, UNESCO, OECD, social cartography

\section{INTRODUCCIÓN}

La inclusión educativa constituye un tema de política educativa de actual abordaje a nivel mundial. Puede definirse como la respuesta a la diversidad desde cauces positivos que se identifica con la participación, logros y supresión de barreras de todos los colectivos involucrados (alumnado, responsables de gestión, profesorado, familia y comunidad) (Plancarte, 2017), proveyendo a los primeros de mayores oportunidades desde el horizonte de logro académico y social (Dainez \& Smolka, 2019; Freda et al., 2017). Desde el enfoque de derechos (Rubio, 2017), interpela a la flexibilidad y a la adaptación, a la responsabilidad y a la educación bajo el principio del respeto a la identidad de todas las personas (Blanco, 2006; Echeita y Ainscow, 2011) a fin de que la totalidad del estudiantado pueda participar y ser tratada como miembros valiosos (Moriña, 2017).

Con el fin de medir la inclusión se han ido considerando una serie de factores concebidos como esenciales (Lawrie et al., 2017). Una propuesta de sistematización de algunos de los indicadores que puede medir la inclusión lo constituye, precisamente, el Index for Inclusion (Booth \& Ainscow, 2011).

Desde el punto de vista de las políticas educativas, Black-Hawkins (2017) también reconoce que la educación inclusiva se ha preocupado más de los procesos amplios políticos, sociales y culturales que en modelar los sistemas educativos, con el fin de hacer una provisión para que todas las personas se eduquen juntas. Esta idea nos remite a analizar cómo las políticas de los organismos internacionales en materia de inclusión educativa están dirigiendo la ortodoxia política a nivel mundial.

Desde estas consideraciones iniciales, el artículo analiza las políticas de inclusión educativa de dos organismos internacionales idiosincrásicos, la Organización para la Cooperación y Desarrollo Económico-OCDE y la Organización de las Naciones Unidas para la Educación, la Ciencia y la Cultura-UNESCO, a fin de demostrar cómo a pesar de los discursos en torno 
a dichas instituciones hablan de homogeneización y de isomorfismo (Zapp \& Ramirez, 2019) en educación, se detectan divergencias que fidelizan a cada uno de ellos con modelos teóricos irreconciliables. Para demostrarlo, se utiliza el estudio de caso a partir de los informes publicados entre 1994 y 2019, a fin de inferir el discurso que sobre la inclusión educativa han mantenido estos dos organismos concebidos como unidades de análisis. En los resultados se adopta la cartografía social, técnica utilizada en educación comparada, para reconocer cuáles son tanto las convergencias como, en especial, las brechas originadas desde la consideración de cada uno de ellos de dos modelos: el discurso del déficit y el modelo de justicia social, respectivamente.

\section{METODOLOGÍA}

La metodología adoptada para abordar esta investigación es de tipo cualitativa. Se basa, en primer lugar, en la selección de fuentes secundarias emitidas por ambos organismos internacionales en las que se aborde la temática vinculada a la inclusión educativa, entre 1994 y 2019. Los informes han sido escogidos de las bases de datos OECD ilibrary para OCDE y UNESDOC para UNESCO. El criterio de selección se ha basado en su naturaleza y alcance global (ni regional ni por unidad de país), realizando a partir de ello una búsqueda de los términos "inclusión" y "educación inclusiva", de la que se obtuvo un total de 20 informes.

Como paso siguiente, se ha escogido el estudio de casos para analizar cada uno de ellos y, para los resultados, la cartografía social como técnica heurística utilizada en educación comparada.

En cuanto a la cartografía social, esta puede ser definida como una propuesta alternativa, dando respuesta desde la metodología al mostrar el intento personal del comparatista para contribuir a la liberación del campo discursivo a través de la tarea de imaginar opciones: desde ellas, se visualizan narrativas teóricas y metodológicas acerca tanto del mundo como de su realidad educativa (Egido y Martínez-Usarralde, 2019). Como Paulston reconoce, supone un nuevo método de identificar las cambiantes percepciones de valores, ideologías y relaciones espaciales, y, a su vez,

Una alianza de la educación y la geografía cultural en el desarrollo de una metodología coherente con la visualización de narraciones en una época en la que la gente se da cuenta de que sus posibilidades y su lugar en el mundo resultan ser bastante distintas de lo que eran unas pocas décadas atrás (Paulston, 2000, p.360). 
Desde esta perspectiva, el investigador ejercerá un rol mediador (Nóvoa, 2018), y se moverá entre metanarrativas diferentes, que tratará de poner en diálogo dentro de un espacio común.

La cartografía social utiliza mapas sociales (Gorostiaga, 2017; Lázaro-Herrero, 2017; Liebman \& Paulston, 1994). Con respecto al valor intrínseco que encierran estos instrumentos, y que se puede observar del propio ejemplo escogido, "los mapas sociales hacen posible una vía de entendimiento de cómo se crean las identidades, y cómo las conexiones entre la espacialidad y la subjetividad son situadas entre las comunidades intelectuales" (Kirby, 1996, p.21). Deben estar, además, abiertos de manera continua al cambio, al remapeo, (traducción del original remaping)" (King, 1999, p.185), a la posibilidad de ampliar los límites de cada modelo y fundirse con el concomitante, significándose unos a otros, y, lo que es más ilustrativo, adoptando nuevas identidades y rasgos.

En esta línea, Paulston (2009) propone las siguientes pautas de análisis a las que se acoge la presente investigación: en primer lugar, elegir el tema o debate que ha de ser mapeado. En segundo lugar, seleccionar aquellos textos que construyen el debate, realizando una lectura en la que se evidencien sus rasgos retóricos. En tercer lugar, identificar las posiciones polares intertextuales que existen en los ejes (los marcos teóricos de los que parten). $\mathrm{Y}$, en cuarto lugar, identificar las comunidades textuales que comparten la forma de ver y comunicar la realidad, para, finalmente, mapear la realidad plasmando las comunidades de conocimiento involucradas en ellas.

En los mapas sociales de la cartografía social la responsabilidad recae en la persona investigadora, que es la que analiza e interpreta tanto los mapas cognitivos de diferentes teorías como los discursos que se manifiestan paralelamente a los mismos. Lo anterior previene sobre una evidencia, y es que "los mapas constituyen un acto de control y poder" (Bateson, 1979, p.3) para el comparatista que estudia los sistemas educativos. Otro de los rasgos axiomáticos es que, alejándose del post-positivismo, esta metodología se adscribe más al postmodernismo, desde el momento en que "esta perspectiva reflexiva de la cartografía social provee de una nueva metodología mejor, capaz de reinscribir y entender la comprensión irónica de la realidad" (Paulston, 2000, p.323).

\section{Caso de OCDE}

¿Desde qué parámetros ha organizado este organismo internacional su visión y misión en torno a la inclusión en materia educativa? Revisando primero, aunque sea superficialmente, los documentos iniciales en torno 
a esta cuestión, los correspondientes a la década de los años noventa nos remiten a una preocupación y toma de decisiones políticas y programáticas en torno a la "discapacidad" como término abarcador de ese momento. Así lo prueban los diferentes informes que tienen como fin analizar la discapacidad en la integración del alumnado dentro de los cauces de escolarización (OECD, 1994, 1995 y 1999), en el sistema educativo post-obligatorio (OECD, 1997), y en la educación superior (OECD, 2003), respectivamente.

Para avanzar en este caso, con la intención también de seguir un relato diacrónico, cabe detenerse en la noción de equidad, pues de la significación que le otorgue dependerá uno de los informes que van a marcar de manera inexcusable las señas idiosincrásicas de este organismo internacional hacia la inclusión (OECD, 2008). En efecto, la OCDE ha publicado varios estudios sobre equidad en educación a lo largo de los últimos decenios y lo ha vinculado de manera firme al constructo de inclusión.

Este binomio se inicia de manera patente con el informe "Diez pasos a la Equidad en educación" (Field, Kuczera, \& Pont, 2007; OECD, 2008). Para el organismo, la equidad se define desde dos dimensiones: la justicia y la inclusión. Mientras la primera se identifica con asegurar que las circunstancias sociales y personales (género, estatus socioeconómico o etnia de origen) no deberían erigirse en obstáculos para conseguir todo el potencial educativo, la inclusión, por su parte, queda definida con "asegurar un mínimo estándar básico de educación para todos" (Field et al., 2007, p.31). Es completada con que "todo el mundo debería ser capaz de leer, escribir y realizar operaciones aritméticas simples" (OECD, 2008, p.2).

La equidad es concebida, por tanto, como el resultado de centrar la atención en ambos parámetros, dado que "implica que todos tenemos las destrezas mínimas necesarias para funcionar en la sociedad actual" (Field et al., 2007, p.48). Y que, por tanto, "un sistema justo e inclusivo que consigue las ventajas de la educación disponibles para todos constituye una de las más poderosas palancas para hacer a la sociedad más equitativa" (OCDE, 2008, p.1). Es cuando introduce una concepción de inclusión ligada a las políticas de transición entre cursos y etapas, la participación de la primera infancia en educación institucionalizada, la adquisición de destrezas básicas y la atención a las deserciones escolares.

El grueso del informe se centra en las estrategias para trabajar la equidad desde la política educativa, diseñando para ello un decálogo de diez pasos estratégicos (figura 1): desde el diseño (estructuras conductivas y trayectorias dentro de los sistemas educativos), las prácticas educativas inclusivas dentro y fuera de la escuela y los recursos y los objetivos prioritarios en los que ha de centrarse. De la primera, el diseño, se critica la 
selección académica temprana y las escuelas comprehensivas. Apuesta con ello por el diseño de una educación inclusiva, a través de un "currículum más flexible y diverso» (OECD, 2008, p.4), con acciones como completar la educación básica y mejorar las transiciones, optar por alternativas equivalentes (formación profesional), servicios de guía y de orientación educativa profesional, líneas ágiles con el mundo laboral y promover escuelas de segunda oportunidad, incluida la educación de personas adultas, además de reconocer el aprendizaje previo adquirido. Respecto a las prácticas, apuesta por el compromiso efectivo con metodologías flexibles en la clase y su coherencia con el consiguiente compromiso también con los hogares: con el fomento de la lectura, por ejemplo, el apoyo a los deberes, el fortalecimiento de cauces de comunicación y la creación de auténticas comunidades de aprendizaje. Finalmente, respecto a recursos, recomienda establecer prioridades por sectores, desde educación infantil a superior, distribuyéndolos por países y regiones, bajo la recomendación tácita de descentralizar, educativamente hablando, a través de la cesión a las escuelas de recursos para programas concretos.

Figura 1

Diez pasos hacia la equidad en educación.

DISEÑO

-1. Limitar la asignación inicial a un programa de estudio y la clasificación por aptitudes académicas y proponer la selección académica.

-2. Controlar la selección de escuela para que se frenen los riesgos a la equidad.

-3. En el nivel de preparatoria (segundo de enseñanza secundaria) ofrecer alternativas atractivas, eliminar los callejones sin salida y evitar la deserción.

PRÁCTICAS:

-5. Identificar y proporcionar ayuda sistemática a quienes se rezaguen en la escuela y reducir el porcentaje de alumnos que repiten el año escolar.

-6. Afianzar los vínculos entre la escuela y la familia ayudando a los padres menos favorecidos a apoyar el aprendizaje de sus hijos.

-7. Responder a la diversidad y prever la inclusión exitosa de los migrantes y las minorías en la educación de las mayorías.

\section{PROVISIÓN DE RECURSOS}

-8. Proporcionar una educación sólida a todos, dando prioridad al servicio en la edad temprana y a la educación básica.

-9. Dirigir recursos a los estudiantes con mayores necesidades.

-10. Fijar objetivos para que haya más equidad, específicamente los relacionados con el logro escolar deficiente y las deserciones.

Fuente: OECD, 2008, p.8

El informe de 2012 parte de la premisa de que "la equidad en educación compensa" (OECD, 2012, p.16). Para ello, analiza los beneficios de la inversión en equidad, al afirmar que superan a los costes tanto para los individuos como para las sociedades y por qué la equidad debería ir, mano con mano, 
con la calidad. Es en este informe donde se alude a que un sistema educativo equitativo puede corregir el efecto de desigualdades sociales y económicas más amplias. En el contexto del aprendizaje, permite a las personas aprovechar al máximo la educación y la formación, independientemente de sus antecedentes (Faubert, 2012; Field et al., 2007). Se desea, en definitiva, insistir en la idea de cómo la equidad en los sistemas educativos puede revertir sobre la competitividad económica y la cohesión social (OECD, 2012, p.24). La calidad educativa va ligada, en este sentido, a cómo esta puede prevenir el fracaso escolar para asegurar una fuerza laboral productiva, mejorar la equidad en la educación para el bienestar social, y cómo invertir en educación temprana mejora la equidad en educación al superar los beneficios a los costes cuando se invierte en completar la educación secundaria superior.

En 2013 se vuelve a incidir, a propósito de la publicación de un informe de discusión, sobre el crecimiento inclusivo y el papel que juega la innovación en él. Se insiste en la idea de que, bajo este concepto, subyace la preocupación de que el crecimiento económico no está reflejando necesariamente una mejora del bienestar ciudadano e incluso puede reducir los ingresos de algunos grupos, en las economías de los países OCDE y, por ende, del resto. De ahí la perentoria necesidad de trabajar una innovación inclusiva según la cual se provee con recursos a grupos de ingresos más bajos y que va más allá de la provisión de servicios. Se pone así de manifiesto que el crecimiento no ha sido hasta ahora inclusivo y que las desigualdades se han convertido en un fenómeno intrínseca y extrínsecamente global, de lo que se desprende que la apuesta por un "crecimiento más justo" está ganando relevancia en las agencias internacionales (OECD, 2013, p.15).

En el informe Equity, Excellence and Inclusiveness in Education. Policy Lessons around the world, a propósito de la celebración del International Summit on the Teaching Profession, el organismo sostiene que

Tanto la equidad como la inclusión significa garantizar que todo el estudiantado alcance al menos un nivel mínimo básico de habilidades. Los sistemas educativos equitativos son justos e inclusivos, y ayudan a su estudiantado a alcanzar su potencial de aprendizaje sin erigir barreras formales o informales ni disminuir las expectativas (OECD, 2014, p.17).

Reconoce también cómo la equidad y la inclusión a menudo se superponen, como cuando la desventaja socioeconómica y el bajo rendimiento convergen en grupos de población específicos. Por ejemplo, la evidencia de PISA indica que un estudiante de 15 años de un hogar relativamente desfavorecido tiene más del doble de probabilidades que un estudiante de una familia acomodada de obtener un puntaje inferior al Nivel 2 en la evaluación de lectura. A partir de estos parámetros, se plantea 
trabajar en desarrollar competencias cualitativas para el profesorado que trabaja con alumnado en escuelas con "the greatest need" (mayores necesidades). También se propone desarrollar líneas políticas que se dirijan a reducir el grado de repetición, la elección temprana, mejorar las que se hallan en posición desaventajada, fortalecer y apoyar el liderazgo escolar, conceder un apoyo sistémico para reestructurar las escuelas, crear un clima de apoyo institucional, utilizar datos para identificar al alumnado en riesgo e intervenir tempranamente, conceder un apoyo continuo y procesual a este estudiantado, explicar las vías diferentes en la organización del tiempo de aprendizaje, sostener altas expectativas utilizando prácticas efectivas de enseñanza y crear conexiones efectivas entre escuela, familia y comunidad.

En Preparing our youth for an inclusive and sustainable world. The OECD PISA Global Competence Framework (2017), se hace referencia a esta denominada "competencia global" como término omnicomprensivo que bien pudiera servir como marco actual en el que insertar la inclusión. Esta es definida en términos de capacidad multidimensional a través de la cual los sujetos pueden examinar cuestiones locales, globales y de naturaleza intercultural, comprendiendo y apreciando las diferentes perspectivas y los puntos de vista mundiales, interactuando de manera exitosa y desde el respeto con los otros, y tomando acciones responsables hacia la sostenibilidad y el bienestar colectivo. Todo lo anterior "para crear oportunidades para todos y avanzar en el respeto compartido para la dignidad humana (...) Juntos, podemos fortalecer la competencia global para conseguir sociedades más inclusivas" (OCDE, 2017, p.3).

Finalmente, en el informe Equity in Education. Breaking Down Barriers to Social Mobility (2018), la equidad vuelve a ocupar el centro de atención, siendo considerada como una característica que no se halla fija en los sistemas educativos: países como Chile, Dinamarca, Alemania, Méjico y Estados Unidos han mejorado este indicador. En este caso, este concepto es revisado desde la mirada a las disparidades socioeconómicas que se producen en los resultados educativos del estudiantado, siendo mayor cuando la relación entre los resultados y su estatus socioeconómico es, a su vez, más débil. De este modo, la equidad significa que, aunque exista cualquier desigualdad entre este colectivo en un sistema escolar, esta no es relativa a su estatus socioeconómico. Resulta notorio, llegados a este punto, destacar cómo se analiza la equidad bajo un prisma vinculado a la resiliencia, aunque no se habla de la inclusión como concepto adherido a la misma. 


\section{Caso de UNESCO}

UNESCO se erige en un organismo internacional que ha nutrido la idea tanto de diversidad como la de inclusión educativa desde el modelo social y humano a lo largo de los decenios y desde su creación en 1945: su vinculación más que indiscutible al desarrollo humano, sostenible y con rostro humano (Martínez-Usarralde y Viana-Orta, 2018) se evidencia en el análisis de contenido de los informes y, tomándolo como caso, se irá extrayendo cómo se revela, especialmente la inclusión educativa, en sus políticas y en su evolución diacrónica.

Bajo estos parámetros, los primeros documentos de UNESCO sobre educación inclusiva hacen referencia al alumnado con necesidades educativas especiales, como lo indican los publicados desde la Conferencia Mundial sobre Necesidades Educativas Especiales de Salamanca (1994). Por su parte, y si bien la inclusión no aparece todavía como concepto, la diversidad es trabajada de manera explícita con el informe emitido en 1996, "Nuestra diversidad creativa", de Pérez de Cuéllar, del que cabe destacar su carácter vanguardista, dado que se incardina en un modelo que se plantea desde la necesidad perentoria de una ética global que se comprometa política y axiológicamente con el pluralismo desde la creatividad y los desafíos de un mundo más mediático.

En 2001 UNESCO creó un Archivo Abierto sobre Educación Inclusiva con el fin de sistematizar experiencias educativas en este ámbito en diferentes países del mundo y de que los responsables en política educativa pudieran examinar la situación en sus propios países y tomar decisiones en esta materia.

Sin embargo, pronto se ampliaría la polisemia de este concepto fecundo. Con el inicio del nuevo decenio y la Cumbre de Dakar (2000) se asienta firmemente una política educativa internacional que marca la idiosincrasia de este organismo y que comenzó el decenio anterior, con la Cumbre de Jomtien (1990) a partir de la cual se forjan los denominados 6 objetivos EFA (Education for All), legitimados por el derecho de todos los niños y niñas a la educación. Marca con ello el inicio, como se afirma en numerosos tratados y textos internacionales y se ha ratificado con instrumentos de naturaleza tanto vinculante como no vinculante, según el cual los Estados tienen la obligación de respetar, proteger y cumplir el derecho de todas las personas a una educación básica de calidad vinculada a la equidad educativa (UNESCO, 2014).

En 2005 ya puede leerse cómo la inclusión es un proceso de dirigir y responder a la diversidad de necesidades de todos los aprendices a través del 
incremento de la participación en el aprendizaje, culturas y comunidades, y reducir la exclusión dentro y como resultado de la educación. Ello requiere "modificar los contenidos, los modelos, las estructuras y las estrategias hacia una visión común que incluya a todos los niños y con la convicción de que es la responsabilidad de los sistemas educar a todos los niños" (UNESCO, 2005, p.25). Introduce, por tanto, un matiz importante que lo vincula a los modelos sociales de la inclusión: la responsabilidad ética y social de las instituciones en esta materia.

En 2008 tiene lugar, a propósito de la Conferencia Internacional de Educación, la cuadragésima octava reunión "La educación inclusiva: el camino hacia el futuro". En ella puede leerse que:

La educación inclusiva es un proceso que entraña la transformación de las escuelas y otros centros de aprendizaje para atender a todos los niños, tanto varones como niñas, a alumnos de minorías étnicas, a los educandos afectados por el VIH y el SIDA y a los discapacitados y con dificultades de aprendizaje. El proceso educativo se lleva a cabo en muchos contextos, tanto formales como no formales, en las familias y en la comunidad en su conjunto. Por consiguiente, la educación inclusiva no es una cuestión marginal, sino que es crucial para lograr una educación de calidad para todos los educandos y para el desarrollo de sociedades más inclusivas (UNESCO, 2008, p.5).

Desde la exégesis que otorga el prisma de los derechos humanos, se percibe un interés creciente en este organismo por el concepto y la práctica de la educación inclusiva, suponiendo que esta tiene por objeto eliminar la exclusión social como consecuencia de actitudes y respuestas a la diversidad en términos de raza, clase social, origen étnico, religión, género y aptitudes. En este sentido, constituye una manera de asegurar "que la Educación Para Todos signifique realmente todos" (UNESCO, 2008, p.7). Además, se erige en un concepto en evolución y se hace una llamada a orientar las políticas y estrategias educativas que se centren tanto en las causas como en las consecuencias de la discriminación, la desigualdad y la exclusión como principales obstáculos en la consecución del marco holístico de los objetivos EFA.

El documento "Fomento de una educación moderna, de calidad e inclusiva" (UNESCO, 2010), llama la atención de que, habida cuenta de la diversidad y la amplia gama de circunstancias existentes en los diversos estados miembros, el objetivo de la inclusión educativa se conseguirá bajo diferentes medios, cuya elección dependerá de las necesidades de cada país. La labor del organismo se identificará con apoyar y garantizar el fomento de sistemas educativos inclusivos y de calidad que tengan en cuenta los 
múltiples vectores capaces de ampliar las oportunidades educativas, en particular para los grupos marginados.

El escenario para conseguir lo que Opertti (2017) apostilla como oportunidad para repensar imaginarios sociales transformados lo constituyen primero los 8 Objetivos de Desarrollo del Milenio-ODM y posteriormente los 17 Objetivos de Desarrollo Sostenible-ODS, pergeñados a partir de la denominada Agenda 2030 y la Declaración de Incheon (UNESCO, 2015). De todos ellos, el ODS 4 exhorta a los países a

garantizar una educación inclusiva y equitativa de calidad y promover oportunidades de aprendizaje durante toda la vida para todos". A través de la agenda, en efecto, se desean abordar las desigualdades educativas vinculadas fundamentalmente al "acceso, participación, procesos y resultados de aprendizaje" (UNESCO, 2015, p.233),

con lo que se traduce en cómo la inclusión se enriquece de acuerdo con el modelo social atribuido. Desde esta atalaya, la agenda aúna esfuerzos para que los países apuesten por políticas de género y se enfoquen al estudiantado tradicionalmente excluido, como el perteneciente a minorías étnicas, lingüísticas, infancia de familias vulnerables económicamente, pueblos indígenas y personas en situación de discapacidad y con necesidades educativas especiales.

Dos años más tarde se publica un documento que marcará un nuevo hito en este organismo, la guía para asegurar la inclusión y la calidad en la educación, destinada a las personas responsables de la formulación de políticas en materia educativa, basada en una serie de herramientas de formación para el desarrollo curricular de la Oficina Internacional de Educación (OIE). Bajo la inclusión y la equidad como principios fundamentales, que deberían "orientar todas las políticas, planes y prácticas educativos, en lugar de ser el foco de una política separada" (UNESCO, 2017, p.18), se idean hasta cuatro dimensiones con el fin de revisar este binomio de política educativa (figura 2): 
Figura 2

Dimensiones del marco de revisión de políticas de inclusión y equidad en UNESCO

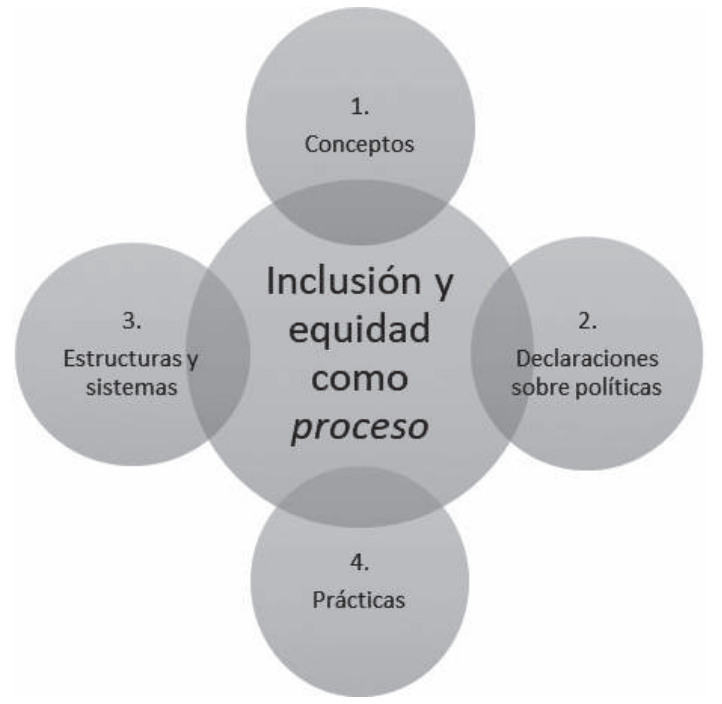

Fuente: elaboración propia a partir de UNESCO, 2017, p.16

Desde esta cuádruple mirada, inclusión y equidad se erigen en principios generales que han de regir todas las políticas, planes, estrategias y prácticas educativas, a fin de atender a todo su estudiantado de manera efectiva y contando con que todos los protagonistas involucrados dentro y fuera de la institución educativa entienden y apoyan los objetivos de la política nacional para promover ambas directrices en educación.

Esta política se acompaña de un plan de evaluación inclusivo del estudiantado a través del cual se trabaja sobre el fomento de un currículum inclusivo que refleje la sociedad a la que se aspira: flexible y adaptable a los diversos conceptos y necesidades "y asegura la equidad y calidad como conceptos gemelos” (IBE-UNESCO, 2017, p.8).

En los últimos informes de UNESCO, ya cercanos al momento actual, el organismo nos recuerda cómo trabaja para construir sociedades de conocimiento inclusivo bajo los principios de equidad, inclusión y calidad recogidos en la arquitectura de los ODS (UNESCO, 2018). Desde este prisma se han editado y difundido tanto el último informe GEM (Global Monitoring Report) que se ha centrado en infancia migrante y desplazada y su relación con la educación (UNESCO, 2019) como el informe de Slee (2018) en el que define el alcance de la educación inclusiva. Este nos sirve, precisamente, para concluir, con la definición que ofrece de la misma, al referirse a: 
Asegurar y garantizar el derecho de todo niño al acceso, presencia, participación y éxito en su escuela local, llamando al barrio a construir su capacidad para eliminar barreras al acceso, presencia, participación y logro a fin de ser capaces de proveer de experiencias educativas excelentes y resultados para toda la infancia y juventud (p.8).

Y esta acción sistemática ha de aplicarse sobre todas las identidades de estudiantado vulnerable: indígenas, niñas, desplazados, minorías étnicas, religiosas o tribales, pobres e infancia en situación de discapacidad; desarrollando, para todos ellos, una comprensión práctica de su exclusión: sus estructuras y sus culturas.

\section{RESULTADOS. CARTOGRAFÍA SOCIAL}

Partiendo de que "la cartografía social ayuda a reconocer patrones y relaciones en contextos espaciales desde lo local hasta lo global, creando con ello un mapa que puede ser comprehensivo y razonablemente seguro, y mantiene un sentido de proporción" (Paulston, 2000, p.318), los resultados son establecidos en la propuesta cartográfica que se presenta a continuación (figura 3), a partir de la cual se inferirán, desde un punto de vista comparado, cuáles son las visiones que sobre inclusión educativa mantienen los dos organismos objeto de estudio, OCDE y UNESCO, ahora considerados como unidades de comparación.

Figura 3

Cartografía social de la inclusión educativa en OCDE y UNESCO

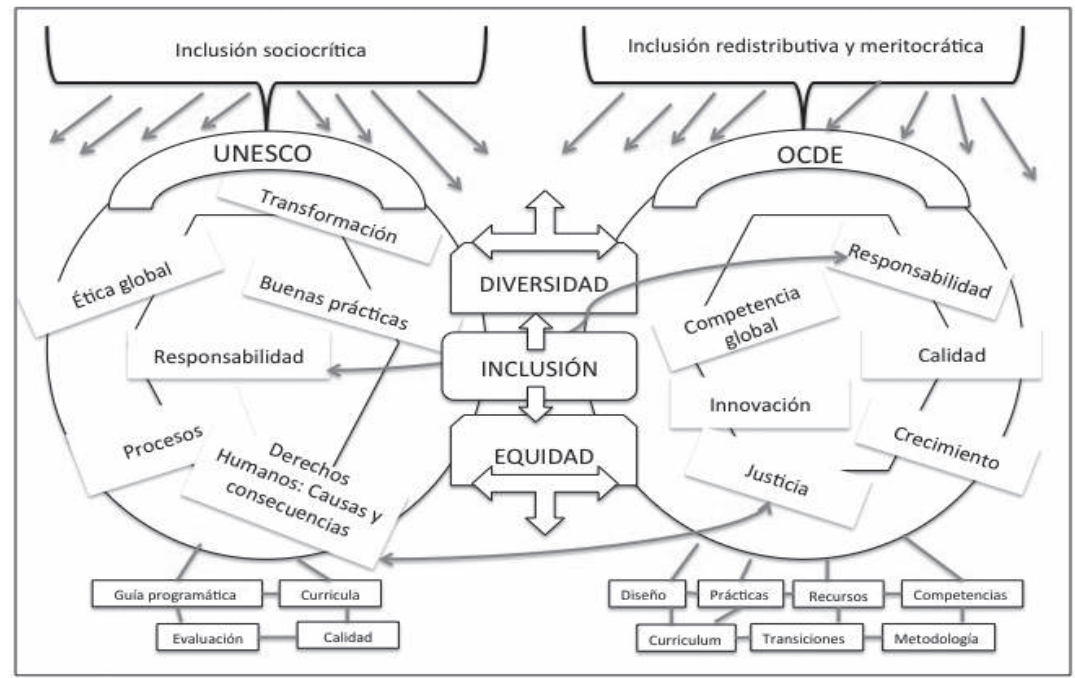

Fuente: elaboración propia 
De acuerdo con las pautas establecidas por Paulston (2009), en primer lugar, la temática escogida ha sido la visión que sobre inclusión educativa detentan ambos organismos.

En la segunda etapa, las ideas-fuerza seleccionadas a partir de los textos que construyen el debate han sido ubicadas en la imagen cartográfica y nos remiten a dos visiones defendidas por dos organismos que, de acuerdo con los casos apuntados, mantienen su propia idiosincrasia:

- Reflejada en la parte derecha, la OCDE se refiere a un concepto de inclusión educativa como parte de un binomio que, junto con la justicia, completa la idea de equidad, tal y como lo prueban los numerosos informes hasta la actualidad, que nos remiten a cómo esta última marida con las disparidades socioeconómicas. Este concepto, además, se enriquece con los sugerentes términos de crecimiento inclusivo e innovación inclusiva ("crecimiento" e "inclusión" en la figura). Pero, volviendo sobre la idea anterior, desde esta óptica, la inclusión queda relegada a erigirse en un concepto-medio y no en concepto-fin, identificándose con la adquisición de estándares básicos (nivel mínimo de habilidades) de educación desde el paradigma de la igualdad de oportunidades y de "calidad" (ir a figura). Especialmente valiosa, para traducir ambos principios, lo constituye la ingeniería socioeducativa generada a fin de trabajar la inclusión educativa desde estrategias que figuran en la parte inferior de la cartografía y que van desde la formación del profesorado de todos los niveles educativos hasta la revisión de diseños, prácticas, recursos, transiciones, metodologías, etc., así como adopción de políticas concretas como la reducción de la repetición y de la elección temprana, adopción de liderazgo y apoyo institucional o atender a los tiempos de aprendizaje; por ejemplo, además de apostar por una promisoria competencia global (visibilizada en la figura) para establecerse en todas las escuelas del mundo y que constituye, en definitiva, su manera de interpretar la "responsabilidad" (también en la figura).

- Mientras, UNESCO, representada en el margen izquierdo de la cartografía, parte de una concepción de "ética global" para entender, dentro del enfoque de "derechos humanos" (mirando por su etiología y su impacto y efectos) y de la "transformación" educativa (reflejados, los tres, en la figura), la obligación de respetar, proteger y cumplir con la inclusión educativa, concepto este del que se recogen "buenas prácticas" (en figura) desde 2001. Trabajando desde una óptica más política las causas y las consecuencias de la desigualdad y la exclusión, la inclusión se vincula a la equidad 
educativa desde sus posibilidades de ampliar las opciones educativas y las oportunidades de acceso, participación, procesos y resultados del alumnado tradicionalmente excluido (intencionalmente destacada en la figura como "procesos"), reflejando de este modo su "responsabilidad" (coincidiendo en la figura con OCDE). También, como en el caso de la OCDE, UNESCO plantea, tal y como recoge la cartografía en su parte inferior, una agenda inclusiva en la que ofrece, en su caso, un plan de formación y una guía programática de políticas, planes, estrategias y prácticas a la que se añade la consideración de currícula inclusiva, y la evaluación.

\section{DISCUSIÓN}

En la discusión se conecta con la tercera etapa de Paulston (2009), en la que se identifican las posiciones polares intertextuales que existen en los ejes. Las posiciones intertextuales de referencia, como muestra la cartografía, nos remiten a dos visiones tangencialmente diferentes desde las que parten ambos organismos, lo que explicaría las divergencias detectadas en su enfoque hacia la inclusión.

En efecto, desde un punto de vista ahora sincrónico, se advierte la convivencia de dos modelos a la hora de definir las políticas de la diversidad y la inclusión educativa (Buenestado-Fernández et al., 2019). Por un lado, el enfoque adoptado por la OCDE que se vincula al desarrollo económico que amerita un sentido redistributivo (igualdad de logros sobre igualdad de proceso) y meritocrático (atención sobre el acceso) de la inclusión (Ahmed, 2012). Se legitiman, por tanto, inclusión y diversidad desde la igualdad de oportunidades, siendo estas representadas equilibradamente entre los diversos colectivos de alumnado y asumiendo la multiculturalidad sobre la dimensión del reconocimiento de las diferencias.

Por otro, el enfoque sociocrítico se identificaría con la postura adoptada desde UNESCO. Este parte de la pedagogía crítica (Mc Arthur, 2010) y del modelo de derechos humanos (Rubio, 2017) desde sus intentos por reconceptualizar la pedagogía inclusiva desde estos referentes y abogar por una "pedagogía post-inclusiva" (Gibson, 2015, p. 876) que evidencia el poder como desventaja en la gestión de grupos educativos (Burke, 2017). Todo lo anterior cristaliza en una concepción bajo el prisma emancipador de la justicia social (Taylor, 2012; Whitburn et al., 2017) y la equidad (Pitman, 2015). En íntima interacción con cuestiones de hegemonía y culturas institucionalizadas, el enfoque acentúa una línea de pedagogía que interpreta el concepto moral de diversidad alineado a la responsabilidad de la sociedad que focaliza su atención en las oportunidades de aprendizaje de los grupos 
más desfavorecidos. En este caso prevalece el interculturalismo e incluso, en sus perspectivas más críticas, "la inclusión transcultural" (Thomsen et al., 2020), destacando los efectos positivos de la diversidad y fomentando el trato diferencial con fines de equidad inclusiva. Se trata, en definitiva, de un enfoque que interpela esta vez a responsabilizar a las instituciones educativas, dado que "la pedagogía crítica necesita ganar fuerza desde diferentes perspectivas, contextos e ideas para seguir contribuyendo a crear espacios públicos, creativos y seguros" (Mc Arthur, 2010, p. 501).

Finalmente, en relación con la cuarta etapa, en la que se identifican las comunidades textuales que comparten la forma de ver y comunicar la realidad, cabe reconocer que las comunidades discursivas erigidas por ambos organismos involucrados en ellas reconocen algunas convergencias que no deben pasarse por alto, señalados con flechas-puente en el gráfico. Esta evidencia nos lleva a pensar cómo, del análisis de las alianzas surgidas en materia multilateral tanto en cooperación como en educación, con alcance mundial, de los que destacan con luz propia Banco Mundial, UNESCO, Unión Europea y OCDE, se percibe una tendencia según la cual estos organismos pierden parte de su idiosincrasia desde que dirimen sus fuerzas dentro de este nuevo pacto mundial: el Banco Mundial habla cada vez más de empoderamiento, mientras que UNESCO, por su parte, lo hace sobre pobreza y subdesarrollo, además de aliarse con sectores privados en materia educativa al ofrecer asistencia y experticia técnica y guía política, con un rol cada vez más rotundo a través de la advocacy (reivindicación de derechos) y de la promoción programática de su ética de valores (Hayhoe \& Mundy, 2008; Martínez-Usarralde y Viana-Orta, 2018).

\section{CONCLUSIONES, LIMITACIONES Y PROSPECTIVA}

En la materia que ahora nos ocupa, la inclusión educativa, en ambos organismos se advierte una evolución de las políticas de "discapacidad" a las políticas de inclusión, y, de acuerdo con ello, en los dos se evidencia también el tránsito del modelo médico de la discapacidad al modelo social de la misma (Borges et al., 2018; Melero et al., 2018; Whitburn et al., 2017). El primero considera a esta como un problema individual y, por tanto, la responsabilidad recae sobre el estudiante. Por su parte, el modelo social postula que la discapacidad no requiere de cura, sino que son las propias instituciones educativas las que, por su parte, generan barreras que influyen en el acceso y participación del estudiantado en situación de discapacidad. La responsabilidad, por tanto, recae principalmente en las últimas y han de ser estas las que reestructuren el currículum, metodologías y clima para que todo el alumnado pueda participar. De ahí que, en la cartografía aportada, 
exista un puente entre ambos términos, considerado como un aspecto convergente.

Las limitaciones que presenta el presente estudio obedecen en buena medida a la línea metodológica adoptada afín a los trabajos de revisión y fundamentación: desde la subjetividad que se desprende del estudio de caso, en cuanto a método cualitativo, se ha tratado de compensar y de demostrar con las citas de los organismos y su retórica del discurso cómo refrendan ambos los modelos inclusión educativa acorde con su visión y su misión. La cartografía aportada ofrece una herramienta exegética para refrendar los hallazgos de los respectivos casos, así como ofrecer una explicación sobre las convergencias y divergencias detectadas.

Como prospectiva, este análisis podría ser complementado en un futuro abundando en la naturaleza comparativa de la investigación y realizando un análisis comparado con indicadores que miden la inclusión desde otros organismos internacionales, como el Banco Mundial o la Unión Europea.

Para concluir, el papel de los organismos internacionales como OCDE y UNESCO consiste fundamentalmente en ofrecer un discurso global que los gobiernos deberán transferir y aplicar posteriormente en función también de los contextos que juegan un papel prioritario. Ambas agencias, por su parte, desde sus respectivos discursos intertextuales, identifican los problemas, los transfiguran en indicadores y organizan su capacidad de resolución ofreciendo recetas político-sociales, en este caso, de naturaleza inclusiva.

\section{NOTA}

— Este artículo forma parte del proyecto EDU2017-82682-R, subvencionado por el Ministerio de Economía, Industria y Competitividad en la convocatoria de Ayudas a Proyectos de I $+\mathrm{D}+\mathrm{I}$ correspondiente al Programa Estatal de Investigación, Desarrollo e Innovación orientada a los Retos de la Sociedad. 


\section{REFERENCIAS BIBLIOGRÁFICAS}

Ahmed, S. (2012). On being included: Racism and diversity in institutional life. Duke University Press.

Bateson, G. (1979). Mente y Naturaleza. Una unidad necesaria. E.P. Dutton.

Black-Hawkins K. (2017). Understanding Inclusive Pedagogy. In V. Plows \& B. Whitburn (Eds.), Inclusive education: Making sense of everyday practice (pp. 13-30). SensePublishers. https://doi. org/10.1007/978-94-6300-866-2_2

Blanco G.R. (2006). La equidad y la inclusión social: uno de los desafíos de la educación y la escuela hoy. REICE. Revista Iberoamericana sobre Calidad, Eficacia y Cambio en Educación, 4(3), 1-15.

Booth, T., \& Ainscow, M. (2002). Index for Inclusion. Developing learning and participation in schools. UNESCO.

Buenestado-Fernández, M., ÁlvarezCastillo J.L., González-González, H., \& Espino-Díaz L. (2019). Evaluating the institutionalisation of diversity outreach in top universities worldwide. PLOS ONE 14(7): e0219525. https:// doi.org/10.1371/journal.pone.0219525

Burke, P.J. (2017). Difference in higher education pedagogies: gender, emotion and shame. Gender and Education, 29(4), 430-444. https://doi. org/10.1080/09540253.2017.1308471

Echeita, G. y Ainscow, M. (2011). La educación inclusiva como derecho. Marco de referencia y pautas de acción para el desarrollo de una revolución pendiente. Tejuelo. Revista de Didáctica de la Lengua y la Literatura, $I V, 26-45$. e https://repositorio.uam.es/ handle/10486/661330

Egido, I., y Martínez-Usarralde, M.J. (2019). La educación comparada hoy: enfoques para una sociedad globalizada. Síntesis.

Dainez, D., \& Smolka, A.L.B. (2019). A função social da escola em discussão, sob a perspectiva da educação inclusiva. Educação e Pesquisa, 45, e187853-e187853. http://dx.doi.org/10.1590/S16784634201945187853.

Faubert, B. (2012). In-school policies and practices for overcoming school failure: A Literature Review for the OECD. OECD Education Working Papers, OECD.

Field, S., Kuczera, M., \& Pont, B. (2007). No More Failures: Ten Steps to Equity in Education. OECD.

Freda, M.F., Rainone, N., Striano, M., \& Valerio, P. (2017). Academic inclusion: a debated and interdisciplinary concept. In M.F Freda, J. GonzálezMonteagudo, \& G. Esposito, (Eds.), Working with Underachieving Students in Higher Education (pp. 35-45), Routledge.

Gibson, S. (2015). When rights are not enough: What is? Moving towards new pedagogy for inclusive education within UK universities. International Journal of Inclusive Education, 19(8), 875-886. https://doi.org/10.1080/09687 599.2012.654987

Gorostiaga, J.M. (2017). Perspectivismo y cartografía social: aportes a la Educación Comparada. Educaçao \& Realidade, 42(3), 877-898. http:// dx.doi.org/10.1590/2175-623665366.

Hayhoe, R. \& Mundy, K. (2008). Introduction to comparative and international education: Why study comparative education. In R. Haynoe, \& K. Mundy (eds.), Comparative 
and international education: Issues for teachers, (pp. 1-21), Canadian Scholars.

IBE-UNESCO (2017). Inclusive Student Assessment. IBE-UNESCO.

King, E. (1999). Education revised for a world in transformation. Comparative Education, 35, 109-117. https://doi. org/10.1080/0305006992791

Kirby, K.M. (1996). Indifferent boundaries: Spatial concepts of human subjectivity, Guilford Press.

Lawrie, G., Marquis, E., Fuller, E., Newman, T., Qiu, M., Nomikoudis, M., ..., \& Van Dam, L. (2017). Moving towards inclusive learning and teaching: A synthesis of recent literature. Teaching \& Learning Inquiry, 5(1), 1-13. http://dx.doi. org/10.20343/teachlearninqu.5.1.3

Lázaro-Herrero, L. (2017). El enfoque socio-histórico en el mapa de la Educación Comparada. Revisión y revalorización de sus postulados. Revista Española de Educación Comparada, 29, 282-296. http://dx.doi. org/10.5944/reec.29.2017.

Liebman, M. \& Paulston, R.G. (1994). Social cartography: A new methodology for comparative studies. Compare, 24(3), 233-245. https://files. eric.ed.gov/fulltext/ED370832.pdf

Martínez-Usarralde, M.J., \& VianaOrta, M.I. (2018). The Life Cycle of UNESCO Education Policies: Fields, Programmes and Strategies. In M. Aki (ed.), Unesco: Current Issues and Challenges, (pp. 25-48), Nova Science Publishers.

McArthur, J. (2010). Achieving social justice within and through higher education: The challenge for critical pedagogy. Teaching in Higher
Education, 15(5), 493-504. https://doi. org/10.1080/13562517.2010.491906

Moriña, A. (2017). Inclusive education in higher education: Challenges and opportunities. European Journal of Special Needs Education, 32(1), 3-17. https://doi.org/10.1080/08856257.2016 .1254964 .

OCDE (2014). Equity, Excellence and Inclusiveness in Education: Policy Lessons from Around de word. OECD.

OECD (1994). The integration of Disabled Children into Mainstream Education: Ambitions, Theories and Practices. OECD.

OECD (1995). Integrating Student with Special Needs Into Mainstream Schools. OECD.

OECD (1999). Inclusive Education at work: Students with Disabilities in Mainstream Schools. OECD.

OECD (2003). Disability in Higher Education. OECD.

OECD (2008). OECD in brief. Ten steps to Equity in Education. OECD Observer. OECD.

OECD (2012). Equity and Quality in Education: Supporting Disadvantaged Students and Schools OECD.

OECD (2013). Innovation and inclusive development. Discussion Report. $O E C D$.

OECD-PISA (2017). Preparing our youth for an inclusive and sustainable world. The OECD PISA global competence framework. OECD.

OECD (2018). Equity in Education. Breaking Down Barriers to Social Mobility. OECD.

Opertti, R. (2017). 15 claves de Análisis para apuntalar la Agenda Educativa 2030. IBE. 
Paulston, R. (2000). Imagining Comparative Education: past, present, future. Compare, 30(3), 353-367. https://doi.org/10.1080/713657467

Paulston, R.G. (2009). Mapping comparative education after postmodernity. In International handbook of comparative education. vol. 22 (pp. 965-990), Springer. https:// doi.org/10.1007/978-1-4020-6403-6_62

Pitman, T. (2015). Unlocking the gates to the peasants: are policies of 'fairness' or 'inclusion' more important for equity in higher education? Cambridge Journal of Education, 45(2), 281293. https://doi.org/10.1080/03057 64X.2014.970514

Plancarte, P.A. (2017). Inclusión educativa y cultura inclusiva. Revista de Educación Inclusiva, 10(2), 213226.

Rubio, V. (2017). Inclusión de personas en situación de discapacidad en educación superior, desde el enfoque de la responsabilidad social, en un contexto de transiciones discursivas respecto del binomio Integración/ Inclusión. Revista Latinoamericana de Educación Inclusiva, 11(2), 199216. http://dx.doi.org/10.4067/S071873782017000200013

Slee, R. - UNESCO-Global Monitoring Report (2018). Inclusion and Education (ED /GEMR/MRT/2018/ T1/1): Defining the Scope of Inclusive Education. Paper commissioned for the 2020 Global Education Monitoring Report, https://unesdoc.unesco.org/ ark:/48223/pf0000265773

Taylor, Y. (Ed.) (2012). Educational Diversity, MacMillan.

Tikly, L.P. (2017). The future of Education for All as a Global Regime of Educational Governance. Comparative
Education Review, 61, 22-57. https:// doi.org/10.1086/689700

Thomsen, B., Muurlink, O., Best, T., Thomsen, J., \& Copeland, L. (2020). Transcultural Development. Human Organization, 79(1), 43-56. https://doi. org/10.17730/0018-7259.79.1.43

UNESCO (1996). Nuestra diversidad creativa. Informe de la Comisión Mundial de Cultura y Desarrollo. https://bit.ly/2CnTUqk

UNESCO (2005). Guidelines for inclusion: Ensuring access to Education for All. UNESCO.

UNESCO (2008). ED/BIE/CONFINTED 48/1. Conferencia Internacional de educación: La educación inclusiva: el camino hacia el futuro, UNESCO.

UNESCO (2009). Directrices sobre politicas de inclusión en la educación, UNESCO.

UNESCO (2010). Consejo Ejecutivo 184 $\mathrm{EX} / 36$. Fomento de una educación moderna, de calidad e inclusiva, UNESCO.

UNESCO (2014). The Right to Education: Law and Policy Review Guidelines, UNESCO.

UNESCO (2015). Educación 2030: Hacia una educación inclusiva y equitativa de calidad y un aprendizaje a lo largo de la vida para todos, World Education Forum.

UNESCO (2017). Guía para asegurar la inclusión y la equidad en la educación, UNESCO.

UNESCO (2018). UNESCO 2017, UNESCO.

UNESCO (2019). Migration, displacement and education. Building bridges, not walls. Global Education Monitoring Report-GEM, UNESCO. 
Whitburn, B., Moss, J., \& O’Mara, J. (2017). Inclusive Education, Subjectivities and the Posts. In V. Plows \& B. Whitburn (Eds.), Inclusive education: Making sense of everyday practice (pp. 31-44). SensePublishers. https://doi.org/10.1007/978-94-6300866-2_2

Zapp, M. \& Ramirez, F.O. (2019). Beyond internationalisation and isomorphism-the construction of a global higher education regime. Comparative Education, 55(4), 473493. https://doi.org/10.1080/03050068 .2019 .1638103 


\section{PERFIL ACADÉMICO Y PROFESIONAL DE LA AUTORA}

María Jesús Martínez Usarralde. ORCID: http://orcid.org/0000-00016777-3399

Profesora del Departamento de Educación Comparada e Historia de la Educación de la Facultad de Filosofía y Ciencias de la Educación de la Universitat de València. Sus intereses de investigación se centran en los temas de Educación Comparada e Internacional, Cooperación para el Desarrollo y educación, inclusión y mediación educativa e intercultural, así como en metodologías de aprendizaje en la universidad como el Aprendizaje Servicio (ApS) y su relación con la Responsabilidad Social Universitaria (RSU). Ha sido investigadora visitante en el Institute of Education (Londres), el Instituto de Investigación y Planificación Educativa-IIPE de UNESCO (Buenos Aires, Argentina) y la Oficina de RSU de la Universidad de Concepción (UDeC) (Chile).

Fecha Recepción del Artículo: 17. Enero. 2020

Fecha Modificación del Artículo: 27. Abril. 2020

Fecha Aceptación del Artículo: 11. Mayo. 2020

Fecha Revisión para Publicación: 18. Junio. 2020 
